\title{
Considerations on PET/MR imaging of carotid plaque inflammation with ${ }^{68} \mathrm{Ga}$-Pentixafor
}

\author{
Jakub Toczek, ${ }^{a}$ and Laurent Riou ${ }^{\mathrm{b}}$ \\ a Cardiovascular Molecular Imaging Laboratory, Section of Cardiovascular Medicine and Yale \\ Cardiovascular Research Center, Yale University School of Medicine, New Haven, CT \\ b Laboratoire Radiopharmaceutiques Biocliniques, Faculté de Médecine de Grenoble, UMR UGA \\ - INSERM U1039, Grenoble, France
}

Received Aug 12, 2020; accepted Aug 12, 2020

doi: $10.1007 / \mathrm{s} 12350-020-02354-3$

\section{See related article, pp. 492-502}

The causal factor driving most of cardiovascular disease is atherosclerosis. Atherosclerotic plaque rupture or erosion may lead to thrombosis and ischemia of the downstream vascular territory. Carotid atherosclerotic plaques can cause transient ischemic attacks and ischemic strokes. Such rupture-prone or vulnerable plaques are characterized by their morphology and inflammation. ${ }^{1}$ While disease presentation has somehow shifted as a consequence of the successful management of risk factors at the population level, ${ }^{2}$ overwhelming evidence of the major the role of inflammation in the pathogenesis of atherosclerosis and cardiovascular events remains, including the demonstration that pharmacological reduction of inflammation decreases cardiovascular events. $^{3}$

Chemokines are defined by their ability to induce directed chemotaxis. The $\mathrm{C}-\mathrm{X}-\mathrm{C}$ Motif Chemokine Receptor 4 (CXCR4) is the main receptor for the C-X-C motif chemokine 12 (CXCL12). The receptor and its ligand play a major role in the homing of hematopoietic cells to the bone marrow and control stem cell retention. CXCR4 was also shown to be upregulated in various types of cancers, in association with cancer cell migration and metastasis, ${ }^{4}$ as well as during inflammation, where CXCR4 drives leucocyte recruitment. ${ }^{5}$ The exact

Reprint requests: Laurent Riou, Laboratoire Radiopharmaceutiques Biocliniques, Faculté de Médecine de Grenoble, UMR UGA INSERM U1039, Grenoble, France; Laurent.Riou@univ-grenoblealpes.fr

J Nucl Cardiol 2022;29:503-5.

$1071-3581 / \$ 34.00$

Copyright (C) 2020 American Society of Nuclear Cardiology. role of CXCL12/CXCR4 axis in atherosclerosis remains to be fully elucidated, with indications of an atheroprotective role primarily associated with an alternative ligand of $\mathrm{CXCR} 4^{5,6}$ and independent from leucocyte migration. The prevalent role in cancer prompted the development of CXCR4 positron emission tomography (PET) imaging using a cyclic pentapeptide analog of CXCL12 radiolabeled with gallium-68, ${ }^{68}$ Ga-pentixafor. ${ }^{7}{ }^{68}$ Ga-pentixafor has been clinically evaluated for tumor imaging as well as for the imaging of inflammation, ${ }^{8}$ including in atherosclerosis. ${ }^{9-14}$ Thus, ${ }^{68} \mathrm{Ga}-$ pentixafor is among the list of PET radiotracers evaluated for molecular imaging of inflammation in human atherosclerosis, along with tracers targeting the $18 \mathrm{kDa}$ translocator protein, somatostatin receptor, folate receptor, and macrophage metabolism, ${ }^{15}$ as well as other tracers targeting processes associated with plaque vulnerability such as apoptosis, hypoxia, neovascularization, and microcalcification.

PET is indeed the imaging modality of choice for molecular imaging, with high tissue penetration and sensitivity. For many applications, PET images need to be coregistered with an alternative imaging modality providing anatomical reference. Computed tomography (CT) or, more recently, magnetic resonance imaging (MRI) are used for this purpose with hybrid PET/CT or PET/MRI systems. While MRI presents some advantages compared to CT, including better soft tissue contrast and absence of ionizing radiation, ${ }^{16}$ it does not directly provide an attenuation map for PET reconstruction. Therefore, an artificial map needs to be generated. Additionally, a major limitation of PET imaging is its relatively limited spatial resolution using current state-of-the-art cameras. The high gallium-68 positron energy is responsible for further resolution degradation compared with fluorine-18, the most commonly used PET radionuclide. The limited spatial 
resolution as compared with the size of the targeted structure leads to partial volume effect, which has a significant impact on image quality and quantification accuracy. Partial volume effect comprises the tissue fraction effect (multiple tissues can be present in a single voxel) and the spillover effect (spill-in and spill-outactivity from an adjacent structure can incorrectly appear in a voxel). Various methods for partial volume correction have been proposed and evaluated over the years. ${ }^{17}$ However, the use of partial volume correction still remains limited to research settings.

The study by Rausch et al. ${ }^{18}$ published in the current issue of the Journal of Nuclear Cardiology, presents the evaluation of the impact of partial volume effect on the quantification of ${ }^{68} \mathrm{Ga}$-pentixafor uptake in carotid atherosclerotic plaques and interrogates an aspect of MR-based attenuation correction. In this retrospective study, a population of patients with lymphoma and imaged with ${ }^{68} \mathrm{Ga}$-pentixafor was analyzed to evaluate tracer uptake in carotid atherosclerotic plaques. The population was selected for presenting at least one region with focal ${ }^{68} \mathrm{Ga}$-pentixafor uptake associated with an anatomical alteration in the carotid arteries, with no mention of neurological symptoms or intervention related to carotid plaques. Carotid plaques (1.7 per patient, 34 in total) were classified according to a modified American Heart Association classification based on MRI features. PET images were reconstructed using a standard MR-based attenuation coefficient map generated from Dixon sequences, which provides 4 classes of tissue: air, fat, lung, and soft tissue. In a subset of data, images were reconstructed with custom attenuation maps including bones (skull and cervical spine). ${ }^{68} \mathrm{Ga}$-pentixafor uptake in the lesion was only minimally underestimated ( $\sim 0.5 \%$ on SUV values) using the standard attenuation correction method compared to the correction including bone mapping. This effect was considered negligible by the authors in any practical sense.

Partial volume correction was performed with a model-based fitting method which operates in projection space and simultaneously estimates the activity concentration within each tissue defined in the volume of interest prior to compensation for partial volume effect. The method was initially developed for single-photon emission computed tomography (SPECT) partial volume correction ${ }^{19}$ and adapted for PET with impressive image enhancement and quantification accuracy in phantom studies. ${ }^{20}$ In the present study, images noncorrected for partial volume effect showed similar $\mathrm{TBR}_{\max }$ values for all type of plaques (average $\mathrm{TBR}_{\max }$, $1.5 \pm 0.3$ ). This result could have been easily attributed to the relatively low number of plaques and the associated low statistical power. Studying a similar population,
Li et al. ${ }^{12}$ failed to show a statistically significant difference in carotid plaque uptake of ${ }^{68} \mathrm{Ga}$-pentixafor between subjects with high and low cardiovascular risk (74 plaques in total), despite a trend in the expected direction (mean $\mathrm{TBR}_{\max }, 1.5 \pm 0.4$ and $1.3 \pm 0.3$ for high and low risk groups, respectively). However, partial volume correction as performed in the study by Rausch et al. dramatically changed the picture. Partial volume correction led to a 2.2-fold increase in $\mathrm{TBR}_{\max }$ values in plaques presumed to be inflammatory based on their morphology (type II, III, IV), while no change was observed in lesions with lower expected inflammation (type I, V). In the most extreme case presented in this study, a relatively small lesion $\left(105 \mathrm{~mm}^{3}\right)$ classified as a type IV plaque presented $\mathrm{TBR}_{\max }$ values of 2.2 and 11.8 before and after partial volume correction, respectively. Intuitively, this can be explained by considering an elevated diffuse activity around a relatively small region and making the assumption that the activity originates primarily from this region. Does this hypothesis reflect biological reality? Did this lesion correspond to a plaque with a high inflammatory cell content and high levels of CXCR4, where tracer-specific uptake exceeded several folds the average lesion identified in the study? Or does this case rather show a potential limit of the partial volume correction method on clinical data? For example, the segmentation of a complex carotid lesion could have been suboptimal and/or the registration between PET and MRI images imperfect because of motion artifact, which would lead to an incorrect estimate of the activities in the segmented structures. In the absence of an independent assessment of tissue activity, it is difficult to conclude. One key assumption of the method used by Rausch et al. is a uniform distribution of the tracer within the segmented tissues. While it is virtually certain that this assumption does not reflect the in vivo situation, it is fair to question whether actual variations in activity within the segmented regions would be large enough to induce nontrivial errors. The authors caution that the absolute quantification of tracer concentrations remains questionable, and reasonably claim that their results only provide an estimate of the influence of partial volume effect.

In practice, the method for partial volume correction used in this study requires the segmentation of the structure of interest and adjacent tissues within a larger volume of interest on the registered anatomical (MRI) image prior to the computation generating the partial volume-corrected images. For a wider adoption, this process needs to be fully integrated and, to some degree, automated-including the segmentation process. Technical advances such as total-body PET cameras with drastically improved resolution and sensitivity are likely to improve the imaging of vascular inflammation. 
Nevertheless, partial volume effect will remain a major confounding factor in PET imaging of small structures. With multiple potentially interesting PET tracers targeting inflammation and other aspect associated with plaque vulnerability, prospective studies are now needed to establish the role of ${ }^{68} \mathrm{Ga}$-pentixafor for the imaging of atherosclerotic plaque inflammation. In this regard, the study by Rausch et al. ${ }^{18}$ provides valuable data to be taken into account for the design of such studies, highlighting the relevance of incorporating partial volume correction for more accurate quantification of tracer activity.

\section{References}

1. Finn AV, Nakano M, Narula J, Kolodgie FD, Virmani R. Concept of vulnerable/unstable plaque. Arterioscler Thromb Vasc Biol 2010;30:1282-92.

2. Pasterkamp G, den Ruijter HM, Libby P. Temporal shifts in clinical presentation and underlying mechanisms of atherosclerotic disease. Nat Rev Cardiol 2017;14:21-9.

3. Ridker PM, Everett BM, Thuren T, MacFadyen JG, Chang WH, Ballantyne $\mathrm{C}$, et al. Antiinflammatory therapy with canakinumab for atherosclerotic disease. N Engl J Med 2017;377:1119-31.

4. Guo F, Wang Y, Liu J, Mok SC, Xue F, Zhang W. CXCL12/ CXCR4: A symbiotic bridge linking cancer cells and their stromal neighbors in oncogenic communication networks. Oncogene 2016;35:816-26.

5. Doring Y, Pawig L, Weber C, Noels H. The CXCL12/CXCR4 chemokine ligand/receptor axis in cardiovascular disease. Front Physiol 2014;5:212.

6. Doring Y, Noels H, van der Vorst EPC, Neideck C, Egea V, Drechsler $\mathrm{M}$ et al. Vascular CXCR4 limits atherosclerosis by maintaining arterial integrity evidence from mouse and human studies. Circulation 2017;136:388-+.

7. Demmer O, Gourni E, Schumacher U, Kessler H, Wester HJ. PET imaging of CXCR4 receptors in cancer by a new optimized ligand. Chemmedchem 2011;6:1789-91.

8. Kircher M, Herhaus P, Schottelius M, Buck AK, Werner RA, Wester HJ, et al. CXCR4-directed theranostics in oncology and inflammation. Ann Nucl Med 2018;32:503-11.

9. Hyafil F, Pelisek J, Laitinen I, Schottelius M, Mohring M, Doring $\mathrm{Y}$, et al. Imaging the cytokine receptor CXCR4 in atherosclerotic plaques with the radiotracer (68)Ga-pentixafor for PET. J Nucl Med 2017;58:499-506.
10. Weiberg D, Thackeray JT, Daum G, Sohns JM, Kropf S, Wester $\mathrm{HJ}$, et al. Clinical molecular imaging of chemokine receptor CXCR4 expression in atherosclerotic plaque using Ga-68-pentixafor PET: Correlation with cardiovascular risk factors and Calcified Plaque Burden. J Nucl Med 2018;59:266-72.

11. Derlin T, Sedding DG, Dutzmann J, Haghikia A, Konig T, Napp $\mathrm{LC}$, et al. Imaging of chemokine receptor CXCR4 expression in culprit and nonculprit coronary atherosclerotic plaque using motion-corrected [Ga-68]pentixafor PET/CT. Eur J Nucl Med Mol 2018;I:1934-44.

12. Li X, Heber D, Leike T, Beitzke D, Lu X, Zhang XL, et al. [68Ga]Pentixafor-PET/MRI for the detection of Chemokine receptor 4 expression in atherosclerotic plaques. Eur J Nucl Med Mol 2018;I:558-66.

13. Li X, Yu W, Wollenweber T, Lu X, Wei YX, Beitzke D, et al. [Ga-68]Pentixafor PET/MR imaging of chemokine receptor 4 expression in the human carotid artery. Eur J Nucl Med Mol 2019;I:1616-25.

14. Kircher M, Tran-Gia J, Kemmer L, Zhang XL, Schirbel A, Werner $\mathrm{RA}$, et al. Imaging Inflammation in atherosclerosis with CXCR4directed Ga-68-pentixafor PET/CT: Correlation with F-18-FDG PET/CT. J Nucl Med 2020;61:751-6.

15. Perez-Medina C, Fayad ZA, Mulder WJM. Atherosclerosis immunoimaging by positron emission tomography. Arterioscl Throm Vas 2020;40:865-73.

16. Saba L, Yuan C, Hatsukami TS, Balu N, Qiao Y, DeMarco JK, et al. Carotid artery wall imaging: Perspective and guidelines from the asnr vessel wall imaging study group and expert consensus recommendations of the american society of neuroradiology. Am J Neuroradiol 2018;39:E9-31.

17. Soret M, Bacharach SL, Buvat I. Partial-volume effect in PET tumor imaging. J Nucl Med 2007;48:932-45.

18. Rausch I, Beitzke D, Li X, Pfaff S, Rasul S, Haug AR et al. Accuracy of PET quantification in [Ga-68]Ga-pentixafor PET/MR imaging of carotid plaques. J Nucl Cardiol 2020.

19. Moore SC, Southekal S, Park MA, McQuaid SJ, Kijewski MF, Muller SP. Improved regional activity quantitation in nuclear medicine using a new approach to correct for tissue partial volume and spillover effects. IEEE Trans Med Imaging 2012;31:405-16.

20. Cal-Gonzalez J, Moore SC, Park MA, Herraiz JL, Vaquero JJ, Desco $\mathrm{M}$, et al. Improved quantification for local regions of interest in preclinical PET imaging. Phys Med Biol 2015;60:712749.

Publisher's Note Springer Nature remains neutral with regard to jurisdictional claims in published maps and institutional affiliations. 